Cita bibliográfica. Olague de la Cruz, J.T., Flores Villanueva, C.A. y Garza Villegas, J.B. (2017). El efecto de la motivación de viaje sobre la satisfacción del turista a través de las dimensiones de la imagen del destino: EL caso del turismo urbano de ocio a Monterrey, México. Investigaciones Turísticas (14), pp. 109-129 http://dx.doi.org/10.14461/INTURI2017.14.06

\title{
El efecto de la motivación de viaje sobre la satisfacción del turista a través de las dimensiones de la imagen de destino: El caso del turismo urbano de ocio a Monterrey, México
}

\section{Effect of travel motivation on tourist satisfaction through the dimensions of destination image: The case of leisure urban tourism in Monterrey, Mexico}

José T. Olague de la Cruz. Universidad Autónoma de Baja California. México. jose.olague@uabc.edu.mx Cesario Armando Flores Villanueva. Universidad Autónoma de Nuevo León. México. cesario.floresvl@uanl.edu.mx Juan Baldemar Garza Villegas. Universidad de Monterrey. México. baldemar.garza@udem.edu

\section{RESUMEN}

El tema de este trabajo es el efecto determinante de la motivación de viaje sobre la satisfacción del turista teniendo como variables mediadoras a los dos componentes de la imagen percibida del destino en un contexto de turistas de ocio a la ciudad de Monterrey, México. A través de un modelo de ecuaciones estructurales por medio de mínimos cuadrados parciales (PLS) fue posible la identificación de los principales indicadores observables de la motivación de viaje de los turistas y obtener evidencia de su papel causal predictivo sobre la satisfacción del turista. En dicha relación, dos componentes principales de la imagen demostraron un efecto mediador para los cuales igualmente quedaron establecidos sus indicadores observables, así como su relación causa - efecto. Con ello se pudo establecer qué aspectos de la motivación son los más importantes para los turistas y vincularlos con la satisfacción del turista y a su vez establecer aquellos aspectos (afectivos y cognitivos) mejor valorados en las percepciones sobre el destino. Finalmente, a modo de conclusión, se emiten recomendaciones generales para la gestión del producto turístico urbano.

Palabras clave: Turismo urbano, satisfacción del turista, motivación de viaje, imagen de destino, PLS, ecuaciones estructurales.

\section{ABSTRACT}

The subject of this research work is the effect of travel motivation on tourist satisfaction with the mediating variables being the two components of the destination's perceived image in a context of leisure tourists to the city of Monterrey, Mexico. Through structural equation modeling by 
partial least squares (PLS) the identification of the main observable indicators of the travel motivation of tourists were identified and thus evidence of its determining role on tourist satisfaction was obtained. In this relationship, two main components of the image showed a mediator effect. The observable indicators of these components were also established as was their cause-effect relationship. Therefore, those aspects of motivation which are the most important for tourists and linked to tourist satisfaction were determined and those aspects (affective and cognitive) best valued in perceptions about the destination were also established. Finally, by way of conclusion, general recommendations for urban tourism product management are provided.

Keywords: Urban tourism, tourist satisfaction, travel motivation, destination image, PLS, structural equations.

\section{INTRODUCCIÓN}

El turismo es un fenómeno presente en casi todos los rincones del planeta. Sus causas y efectos han sido abordados desde un creciente número de disciplinas debido al impacto que produce como potenciador del progreso socioeconómico, el empleo y el desarrollo de infraestructura. Hoy día, la gran mayoría de naciones del orbe impulsan la actividad turística para beneficiarse y por tanto se integran a un ambiente competitivo que crece cada año. Entre las muchas formas que toma el turismo se encuentra el llevado a cabo en aglomeraciones urbanas y que se ha denominado como turismo urbano (Organización Mundial del Turismo, 2014). Un gran número de ciudades destinan inversiones crecientes a la creación de ventajas competitivas para tal efecto. En el caso mexicano, uno de los principales destinos turísticos del mundo, la ciudad de Monterrey ocupa un lugar especial como una de las ciudades más visitadas en el país, por lo que se ha convertido en referente sobre el desarrollo del turismo hacia ciudades.

El desarrollo de estrategias para la segmentación, desarrollo de productos, posicionamiento o bien los esfuerzos promocionales realizados por los destinos turísticos implican el uso de enormes recursos económicos. La motivación de viaje y la imagen de destino han sido identificadas en la literatura como directa y positivamente relacionadas entre sí (Lubbe, 1998; Baloglu y McCleary, 1999; Beerli y Martín, 2004; San Martín y Rodríguez, 2008; Stabler, 1988; Chon, 1989) y también como antecedentes directos de la satisfacción de los turistas (Yoon y Uysal, 2005; Lee, Lee, y Wicks, 2004; Battour, Ismail, y Battor, 2014; Bigné, Sánchez, y Sánchez, 2001; Medina, Rey, y Rufín, 2010; Lee T. H., 2009). Asimismo ha recibido atención de los investigadores la relación determinante entre las dimensiones cognitiva y afectiva de la imagen de los destinos (Baloglu y McCleary, 1999; Matos, Mendes, y Valle, 2012; Beerli y Martín, 2004). Todas esas variables pueden adquirir estructuras complejas por lo que se vuelve necesario dimensionarlas adecuadamente antes de tomar decisiones de mercadotecnia de destino.

En la literatura reciente, se ha abordado problemas de este tipo desde el análisis causal. El resultado ha sido la propuesta exitosa de modelos estructurales (Nunkoo y Ramkissoon, 2012). En los últimos años la técnica de ajuste vía mínimos cuadrados parciales destaca como una opción adecuada para identificar componentes que explican la relación entre variables fundamentales en el proceso de conducta del consumidor y su decisión de compra (Varasteh, Marzuki, y Rasoolimanesh, 2015; Wu, Raab, Chang, y Krishen, 2016; Gómez, Molina, y Esteban, 2013). Por lo 
anterior el objetivo de este trabajo fue probar un modelo causal predictivo capaz de explicar la satisfacción del turista en función de la motivación de viaje a través de las dimensiones de la imagen del destino a través del método de ecuaciones estructurales por mínimos cuadrados parciales. Su utilización para abordar el caso de Monterrey como destino turístico permite obtener información útil en la toma de decisiones para orientar el uso de recursos destinados a la promoción de la actividad turística e incidir en el positivo desarrollo económico de su comunidad.

\subsection{El turismo urbano en la actualidad}

El turismo nunca ha sido un fenómeno ajeno a las ciudades. Ya desde la antigüedad la gente ha emprendido viajes con el objeto de conocer el patrimonio contenido en centros urbanos o bien experimentar sus modos de vida y otras expresiones culturales. Las grandes ciudades europeas de Francia, Italia o Alemania figuraban ya entre los puntos a visitar como parte del Grand Tour que las clases privilegiadas solían realizar como parte de su educación desde los siglos XVII al XIX (Guerrero González y Ramos Mendoza, 2011). En la actualidad, el turismo hacia las grandes ciudades continúa su crecimiento sostenido. El Internationale Tourismus-Börse (ITB) World Travel Trends Report (diciembre 2014) lo identificaba como el segmento de mayor crecimiento con una tasa del $58 \%$ de crecimiento en los cinco años inmediatos anteriores. En la actualidad la cuota de mercado de los viajes a ciudad ronda cerca del $20 \%$ y ciudades como París, Londres y Nueva York encabezan la lista de los destinos más visitados.

No es sorpresa que las ciudades se encuentren entre las preferidas de los turistas para visitar en su tiempo libre. Los turistas, al ser usuarios de la mayoría de los servicios e instalaciones de las ciudades como entes multifuncionales son absorbidos a la perfección por la dinámica urbana (Ashworth, 2012). Este fenómeno ha dado origen a una tipología turística diferenciada y caracterizada por el involucramiento del turista en múltiples actividades de compleja distribución sin el predominio de ninguna en particular y donde el único signo distintivo es su contexto urbano (Xiao, 2007). Ciertos elementos estructurales que convierten a las urbes en destinos ideales (Violier y Zárate Martín, 2007):

1. La existencia de cultura, monumentos, fenómenos artísticos, paisajes u otros aspectos morfológicos.

2. La concentración de actividades profesionales, negocios, convenciones, ferias y otros espacios de intercambio

3. Suelen ser la sede de servicios administrativos y de comercialización de bienes y servicios.

4. Son espacios para los grandes acontecimientos deportivos y cuentan con instalaciones para el ocio.

Esta condición ha sido aprovechada por muchas ciudades para mejorar sus condiciones económicas y emprender procesos de regeneración. Kotler, Heider y Rein (1994) señalaron el fomento de la actividad turística por parte de muchas ciudades en los Estados Unidos para superar escenarios de crisis resultado de fuerzas internas y externas desfavorables. Dichas crisis son resultado de la evolución globalizadora de la economía y que obliga a las ciudades, en muchas ocasiones, a buscar estrategias centradas en la atracción de visitantes que produce efectos 
equiparables a un aumento de las exportaciones, fomenta la regeneración urbana y promueve el empleo (Rogerson, 2013).

En el entorno actual donde las urbes compiten por la atracción de visitantes es importante el enfoque estratégico en el cual las ciudades deberían atender a cuatro aspectos: características físicas de la ciudad, elementos de la comunidad urbana, gestión de la oferta y un marketing orientado al visitante (Paskaleva-Shapira, 2007) que les permitirán la atracción de eventos y segmentos de interés especial caracterizados por su rentabilidad. Dicho enfoque no debe dejar de lado su enfoque centrado en la comunidad local y su calidad de vida (Law, 1992) y que entienda al turismo como fuerza transformadora (Galdini, 2007).

\subsection{El turismo urbano en México: Especial referencia a la ciudad de Monterrey}

México se encuentra en la actualidad ubicado entre los principales destinos internacionales alcanzando una cifra superior a los de veinticuatro millones de turistas internacionales que, sumados a los poco más de 52 millones de turistas nacionales (DATATUR, 2014) permiten dimensionar la importancia de la actividad turística en el país. Para 2013, los diez mayores centros urbanos nacionales registraban, en términos generales, una tendencia al alza de la actividad turística (Tabla 1). La Ciudad de México, D.F. tuvo una afluencia de 12,022,748 de visitantes, mientras que le siguieron: Guadalajara con 3,108,097; Puebla con 1,989,514 de visitantes, Monterrey con 1,688,110 y León con 1,290,183. (Figura 1).

Tabla 1. Llegadas de turistas en los principales destinos urbanos de México (2009 - 2013) (número de visitantes)

\begin{tabular}{lccccc}
\hline & 2009 & 2010 & 2011 & 2012 & 2013 \\
\hline Distrito Federal & $9,179,626$ & $9,899,693$ & $11,206,116$ & $11,859,457$ & $12,022,748$ \\
Guadalajara & $2,260,800$ & $2,512,252$ & $2,712,936$ & $2,974,090$ & $3,108,097$ \\
Monterrey & $1,645,626$ & $1,450,869$ & $1,388,139$ & $1,542,357$ & $1,688,110$ \\
Puebla & $1,170,467$ & $1,309,125$ & $1,497,743$ & $1,718,335$ & $1,989,514$ \\
Toluca & 532,222 & 603,918 & 671,994 & 553,170 & 574,870 \\
Tijuana & 720,713 & 829,480 & 851,723 & 896,873 & 862,557 \\
León & 921,935 & $1,076,609$ & $1,138,702$ & $1,233,018$ & $1,290,183$ \\
Ciudad Juárez & 453,110 & 399,717 & 406,149 & 481,834 & 508,796 \\
Querétaro & 810,863 & 930,110 & 977,173 & $1,060,640$ & $1,063,723$ \\
San Luis Potosí & 579,160 & 726,164 & 712,777 & 631,701 & 585,582 \\
Mérida & 916,214 & 953,254 & $1,044,536$ & $1,062,259$ & $1,221,418$ \\
\hline
\end{tabular}
Fuente: Elaboración propia en base a DATATUR (2014).

Monterrey es la capital del estado de Nuevo León y una de las principales urbes de la República Mexicana. Para el año del censo 2010, la ciudad era el hogar de 1,135,550 habitantes y de 4,106,054 habitantes si se considera también su área metropolitana (INEGI, 2010). El estado de Nuevo León, donde su ubica dicha connurbación contribuye con un $7.5 \%$ del producto interno bruto (PIB) de la nación por lo que la ciudad ocupa un lugar destacado como centro de negocios, industria e intercambio de servicios. Dada su dotación de modernas infraestructuras Monterrey era ya también contado entre los destinos turísticos de mayor atracción desde principios del siglo XXI. En su estudio geográfico Propin Frejomil y Sánchez Crispín (2002) identificaron la pertenencia 
de la ciudad a dos regiones turísticas bien definidas, una microrregión formada junto a la ciudad de Saltillo y de forma más amplia es parte de la macrorregión turística fronteriza del Norte de México. El entorno geográfico de Monterrey se caracteriza por la confluencia de varios sistemas orográficos e hidrográficos que aportan una serie de recursos naturales a su inventario de recursos turísticos. Asimismo la historia de la ciudad, como uno de los primeros centros industriales del país, le ha legado una herencia cultural e histórica que en la opinión de algunos asegura la presencia sostenida de turistas dada la mezcla natural - cultural de su oferta turística y la presencia de buenos servicios de alojamiento, restauración y entretenimiento (Tabla 2) (Sánchez Crispín, López López y Propin Frejomil, 2005).

Figura 1. Evolución de llegadas de turistas en los principales destinos urbanos de México.

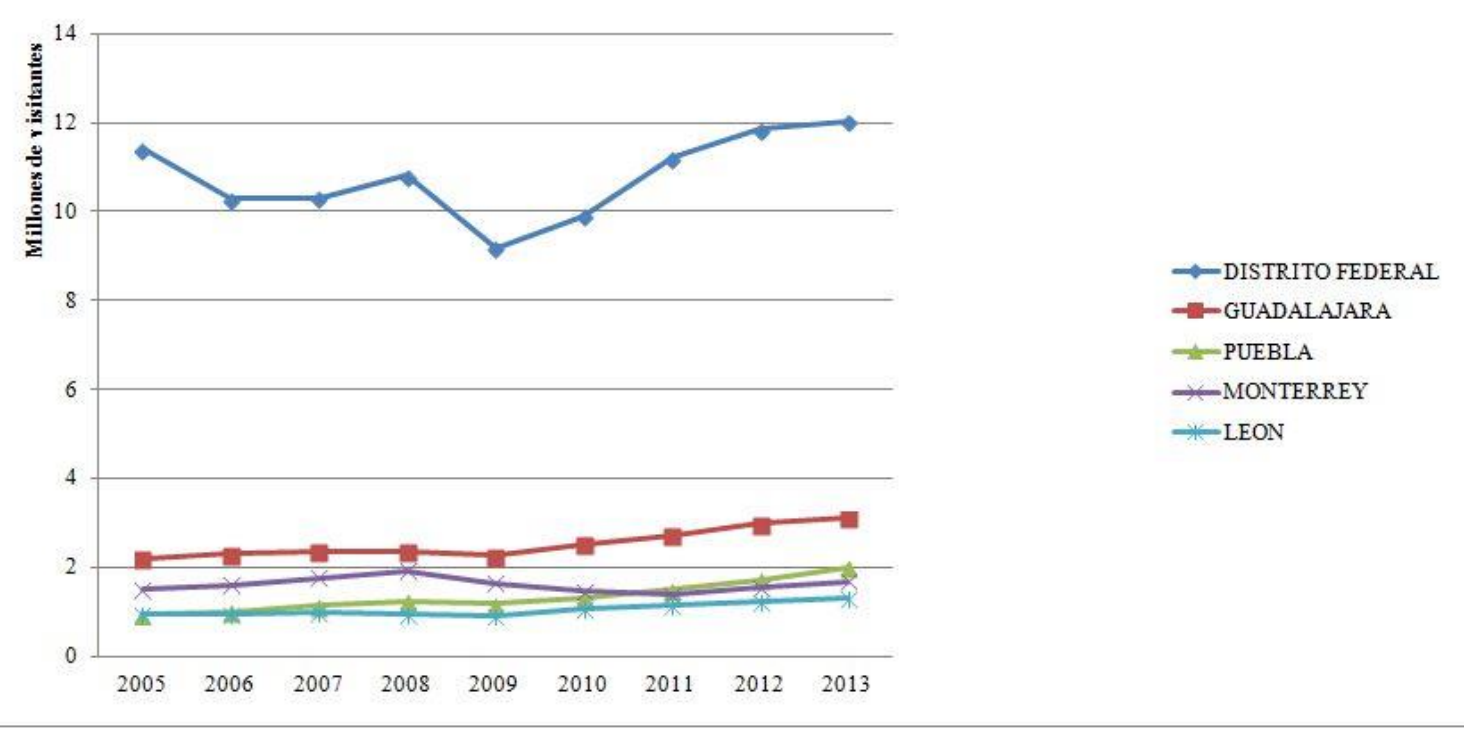

Fuente: Elaboración propia en base a DATATUR (2014). 
Tabla 2 . Recursos naturales, culturales - históricos y urbanos de la estructura territorial del turismo en la ciudad de Monterrey y área metropolitana

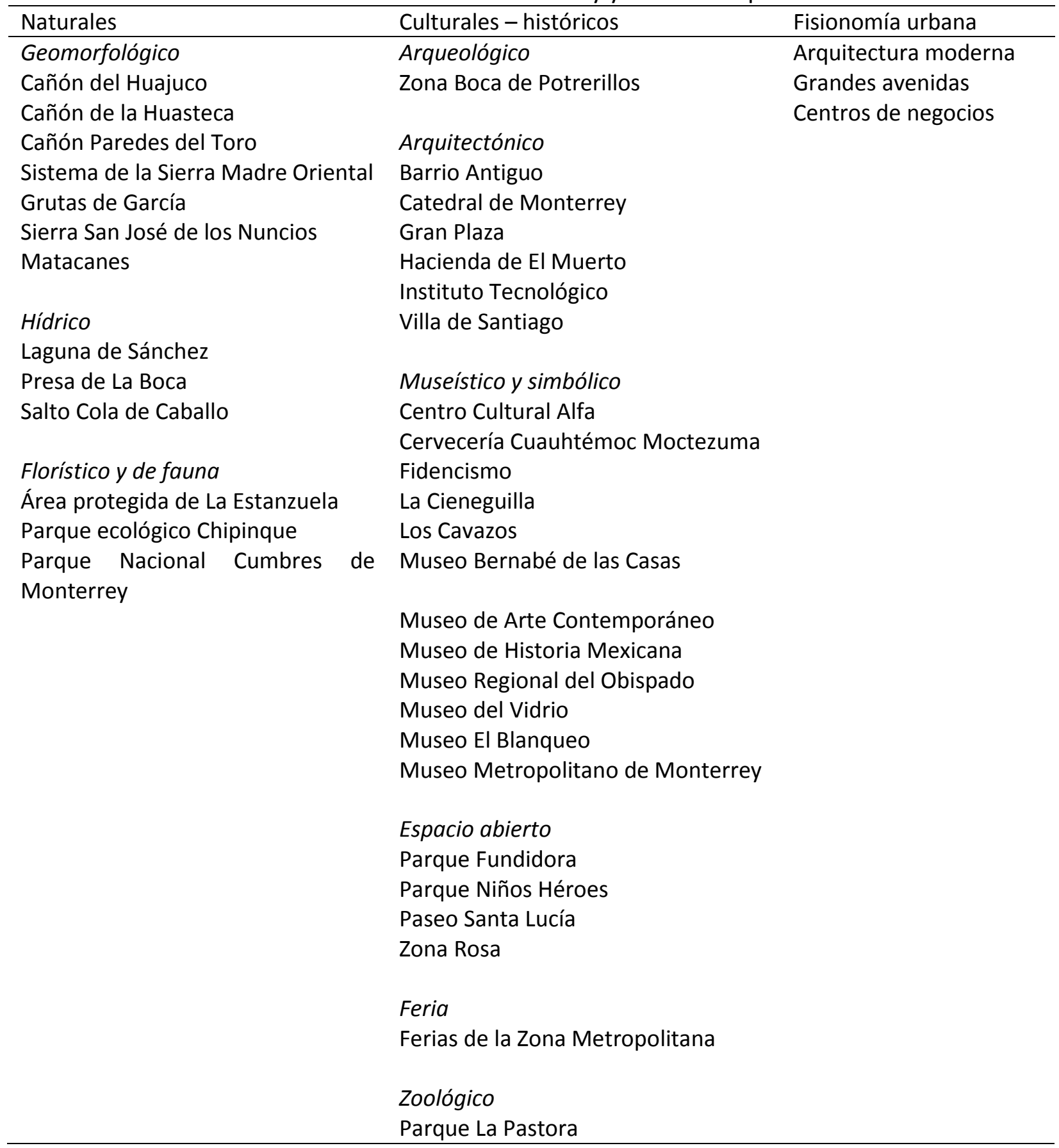
Fuente: Elaboración propia en base a Sánchez Crispín, López López y Propin Frejomil (2005).

De acuerdo con Propin Frejomil y Sánchez Crispín (2007) Monterrey es visitada mayormente por turistas nacionales si bien posee una corriente discreta de turismo extranjero presente en la ciudad como resultado directo de las actividades industriales y comerciales que constituyen la vocación de la urbe. De acuerdo con la Secretaría de Turismo (DATATUR, 2014) Monterrey registró en 2013 una afluencia turística de casi 1.7 millones de turistas, en su mayoría 
provenientes de otros puntos de la República Mexicana. La cantidad de turistas extranjeros fue de casi 305 mil visitantes. Dichos flujos generaron una ocupación de casi seis mil quinientos cuartos (54.22\% promedio anual). Los turistas nacionales y extranjeros permanecieron en la ciudad un promedio de dos noches. La derrama económica reportada fue superior a los 680 millones de dólares estadounidenses.

\section{FUNDAMENTOS TEÓRICOS Y CONCEPTUALES DE LAS VARIABLES DE ESTUDIO}

\subsection{La satisfacción del consumidor - turista: importancia y fundamentación}

El quehacer de negocios tiene en la búsqueda de la satisfacción del turista uno de sus objetivos instrumentales, dada la correlación positiva entre satisfacción, lealtad del consumidor y desempeño económico y financiero de la empresa (Asher, 1989). Destacan en este sentido las evidencias aportadas por Anderson y Sullivan (1993) y Rust y Zahorik (1993) sobre la relación entre satisfacción del cliente y la tasa de retención de los mismos. Tambien los estudios de Anderson (1996); Huber, Herrmann y Wricke (2001) y Homburg, Koschate y Hoyer (2005) sobre como los clientes satisfechos son menos sensibles al precio del producto. Finalmente las aportaciones de Hallowell (1996) y de Anderson, Fornell y Mazvancheryl (2004) sugieren el papel determinante de la satisfacción de los clientes sobre la rentabilidad del negocio y el valor del accionista, respectivamente.

Los anteriores hallazgos encuentran eco en el contexto de los destinos turísticos. La Organización Mundial del Turismo (1985) apunta a la satisfacción del turista como el bienestar y placer obtenido de la interacción entre experiencia y expectativas previas del turista en el destino. Asumida esta idea, el destino turístico sería entonces un producto evaluable en su capacidad de proveer satisfacción al turista. (Dmitrovic et al., 2009). Los trabajos de Phetvaroon, Rachjaibun y Promsivapallop (2006) y Khaki y Sahaf (2011), con resultados similares a las investigaciones anteriormente señaladas en el campo de los negocios, sugieren a la satisfacción del turista como la mejor medida de evaluación del desempeño de un destino.

\subsection{Relación entre las variables de estudio: antecedentes teóricos y conceptuales}

La motivación para viajar tiene su raíz en el estado de tensión y desequilibrio que se produce a nivel psicológico en el turista por efecto de necesidades y deseos (Crompton, 1979). Su estudio se ha abordado desde varios enfoques. Uno de ellos desde la teoría de la jerarquía de las necesidades de Maslow (1970) con base en la cual Pearce $(1988 ; 1993)$ formuló inicialmente un modelo de peldaños de la carrera de viaje según el cual las motivaciones del turista van cambiando de acuerdo a su experiencia acumulada y de forma jerarquizada en cinco niveles: relajación, seguridad, relaciones sociales, autoestima y autorrealización. Esta idea se modificó en una revisión posterior (Pearce, 2005, p. 79) en un modelo de patrones de la carrera de viaje donde las motivaciones del turista coexisten traslapadas más que jerárquicamente: en el núcleo las motivaciones de búsqueda de novedad, escape / relajación y relaciones sociales, en una capa menos importante motivos orientados hacia sí mismo (como la autorrealización) seguidos por motivaciones más externas (como la naturaleza o el involucramiento y al final motivos menos importantes como la nostalgia y el aislamiento (Huang y Hsu, 2009). 
En otra línea, de interés para este trabajo, Iso - Ahola (1982) propuso una interpretación dicotómica de la motivación de viaje donde todo turista tiene razones de "escape" (de la dinámica cotidiana) o "búsqueda" (de recompensa psicológica a través del viaje). Dicha visión, a decir de Crompton y McKay (1997), sería un refinamiento de la teoría de impulso - atracción (Dann, 1977; Crompton, 1979). Dicha teoría fue aplicada al comportamiento del turista en el trabajo de Gnoth (1997) donde la motivación de viaje puede provenir desde dos ámbitos: los impulsos (Hull, 1943) y las expectativas (Vroom, 1964). Por un lado, internamente, el turista experimenta impulsos que lo llevan a buscar una serie de objetivos con los cuales reducir su impulso. Por el otro, hay factores que atraen al turista a partir de su conocimiento previo de objetos con ciertos atributos. Los impulsos se manifiestan en necesidades y deseos y los atributos de cada destino generan expectativas. En la literatura anglosajona se conoce a ambos componentes como push (impulso) y pull (atracción). La teoría de impulso - atracción es útil cuando se requiere identificar y medir las fuerzas que condicionan la elección de un destino (Chan y Baum, 2007; Castaño Blanco, Moreno Sáez, García Dauder, y Crego Díaz, 2003).

La motivación del turista ha encontrado interés en la investigación del comportamiento del consumidor por su capacidad determinante sobre otras variables relacionadas al consumo turístico. Diversos trabajos han aportado evidencia de la influencia de las motivaciones del turista sobre: la percepción de la factibilidad de viaje (Kim y Chalip, 2004); las actitudes del turista hacia el destino y la elección de éste (Nyaupane, Paris, y Teye, 2011; Bansalt y Eiselt, 2004; Anderson, Juaneda, y Sastre, 2009) y el grado de involucramiento del turista en su experiencia en el destino (Kim K. , 2008; Mehmetoglu y Normann, 2013; Chen, Li, y Chen, 2013; Wong y Tang, 2016). En este cúmulo de investigaciones destacan aquellas que sitúan a la motivación de viaje como antecedente de la satisfacción del turista (Yoon y Uysal, 2005; Smith, Costello, y Muenchen, 2010; Correia, Kozak, y Ferradeira, 2013; Devesa, Laguna, y Palacios, 2010; Lee T. H., 2009; Meng, Tepanon, y Uysal, 2008) y aquellas que evidencian su papel determinante sobre la imagen percibida del destino (Lubbe, 1998; Baloglu y McCleary, 1999; Beerli y Martín, 2004; San Martín y Rodríguez, 2008; Stabler, 1988; Chon, 1989).

La capacidad moderadora de la motivación de viaje ha sido también objeto de interés de diversos trabajos que han evidenciado su influencia potenciadora o inhibidora sobre la relación entre variables de la conducta de consumo turístico, al menos de forma parcial. Por ejemplo sobre la relación que guardan variables como la elección de destino y el precio que el turista está dispuesto a pagar o la distancia que está dispuesto a recorrer (Nicolau y Más, 2006). También en la relación entre los rasgos de personalidad y la satisfacción del turista con el consumo de alimentos típicos (Ji, Wong, Eves, y Scarles, 2016). Asimismo la influencia moderadora de la motivación aparece en la relación entre las creencias ambientales y las actitudes ecoturísticas (Hultman, Kazeminia, y Ghasemi, 2015). Es de señalarse que tales efectos moderadores dependen del tipo de motivación que impulse al turista.

Con respecto a la imagen del destino, en ella convergen todas las opiniones, pensamientos, sentimientos, visualizaciones e intenciones del turista (Tasci, Gartner y Cavusgil, 2007). La imagen del destino es uno de los antecedentes que orientan la toma de decisión de viaje y a través de un proceso repetitivo el turista va modificando la imagen que posee de un lugar agregando información obtenida de la experiencia (orgánica) o que le es suministrada 
deliberadamente (inducida) (Gunn, 1988). La imagen del destino puede abordarse y medirse de forma holística tanto como por atributos y en ella se encuentran aspectos afectivos intangibles (sentimientos y emociones) tanto como cognitivos y tangibles (Echtner y Ritchie, 2003). Dichos componentes guardan relaciones de causalidad con los componentes cognitivos determinando a los afectivos y estos a su vez actuando como causa de la imagen holística del destino (Baloglu y McCleary, 1999; Matos, Mendes, y Valle, 2012; Beerli y Martín, 2004).

Asimismo la imagen del destino ha sido identificada como antecedente de la satisfacción del turista por diferentes autores (Bigné, Sánchez, y Sánchez, 2001; Lee T. H., 2009; Wang y Hsu, 2010; Faullant, Matzler, y Füller, 2008; Medina, Rey, y Rufín, 2010). Primero por su papel decisivo en la selección del destino de viaje ya que aquellos sitios mejor posicionados en la mente del turista tienen siempre mejores posibilidades de ser seleccionados. De la misma forma la imagen condiciona las expectativas que el turista tiene sobre el destino y la posterior (des)confirmación de éstas da origen a los juicios de satisfacción o insatisfacción.

\subsection{Hipótesis}

La orientación de este trabajo es de tipo cuantitativo a la vez que correlacional y explicativo, ya que a través de datos cuantificables se pretende identificar relaciones de causalidad entre variables. Se plantearon entonces las siguientes hipótesis de investigación en el contexto de los turistas de ocio al destino urbano de Monterrey, México (Tabla 3):

Tabla 3. Hipótesis de investigación

\begin{tabular}{llc}
\hline & Relación & Efecto propuesto \\
\hline $\mathrm{H}_{1}$ & Motivación de Viaje $\rightarrow$ Imagen Afectiva & + \\
$\mathrm{H}_{2}$ & Motivación de Viaje $\rightarrow$ Imagen Cognitiva & + \\
$\mathrm{H}_{3}$ & Imagen Cognitiva $\rightarrow$ Imagen Afectiva & + \\
$\mathrm{H}_{4}$ & Imagen Cognitiva $\rightarrow$ Satisfacción del Turista & + \\
$\mathrm{H}_{5}$ & Imagen Afectiva $\rightarrow$ Satisfacción del Turista & + \\
\hline
\end{tabular}

Elaboración propia

\section{METOdOLOGÍA}

Tomando como base trabajos previos se elaboró un instrumento capaz de medir de forma válida y confiable las variables estudiadas, se adicionaron también ítems para la medición de aspectos sociodemográficos y de hábitos de viaje de la población de estudio. Se utilizaron como base, en la elaboración de los ítems del cuestionario referentes a la satisfacción del turista, los trabajos de Bigné et al. (2001), Beerli y Martín (2004), Yoon y Uysal (2005), Baloglu et al. (2004), Chen y Tsai (2007), Chi y Qu (2008), Chung y Petrick (2012) y Wang y Hsu (2010). Para la medición de la imagen del destino, los trabajos de Beerli y Martín (2004), Wang y Qu (2006), Buhalis (2000), Edwards et al. (2008), Enright y Newton (2004), Bernini y Cagnone (2012), Baloglu et al. (2004), Dwyer y Kim (2003), Fallon y Schofield (2006). Con respecto a los ítems relativos a la motivación se recurrió a los trabajos de Yoon y Uysal (2005), Meng et al. (2008), Lee (2009), Correia et al. (2013), Lee y Hsu (2013) y Kao et al. (2008). El desarrollo del cuestionario involucró un test previo para asegurar la validez y confiabilidad del mismo, en el que participaron 60 turistas de ocio a la ciudad aprovechando el fin de semana largo del feriado del 17 de marzo de 2014. 
El instrumento final quedó constituido por 1 único ítem para medir la satisfacción del turista de forma holística, 20 ítems para medir la imagen del destino con 4 ítems de diferencial semántico de cinco puntos para medir aspectos afectivos del destino y 16 ítems de tipo Likert - 5 para medir aspectos cognitivos y 12 ítems para medir la motivación de viaje tipo Likert, seis de ellos para medir motivaciones de impulso (push) y seis para medir motivaciones de atracción (pull). Se usó igualmente una escala de tipo Likert -5.

Este trabajo tuvo como metodología central para prueba de hipótesis el uso de ecuaciones estructurales ajustadas por mínimos cuadrados parciales (Partial Least Squares - PLS por sus siglas en inglés). Las ecuaciones estructurales son una técnica ampliamente usada en el contraste de modelos causales. Esta técnica permite construir modelos de investigación a través establecimiento de variables latentes e indicadores basados en conceptos teóricos y conceptos empíricos respectivamente que se relacionan a través de hipótesis (Henlein y Kaplan, 2004). Se utilizó el software estadístico Smart PLS 2.0 y la metodología se basa en la expuesta por Leyva y Olague (2014).

Se realizó una serie de entrevistas estructuradas (encuesta) durante el periodo vacacional de Semana Santa en puntos de afluencia turística en la ciudad de Monterrey, desde el 12 hasta el 29 de abril de 2014. Se seleccionó una muestra por conveniencia aprovechando los puntos de Información Turística del Gobierno del Estado. Se filtró a los participantes con cuatro criterios: debían ser mayores de edad, tener el castellano como lengua materna, que hubieran pernoctado por lo menos una noche en el destino y que su propósito principal de viaje hubiera sido el ocio. Quedó establecido para el estudio un tamaño de muestra bajo los criterios de $95 \%$ de nivel de confianza y un $5 \%$ de margen de error (379 casos válidos).

\section{RESULTADOS Y DISCUSIÓN}

\subsection{Descripción de la muestra}

Se aplicaron en total 1296 entrevistas estructuradas. Luego de concluido un proceso de depuración para descartar cuestionarios inconsistentes o incompletos la muestra se redujo a 649 casos válidos. El perfil socio - demográfico se midió por género (masculino, 48.7\%; femenino 49.2\%, no contestó (N/C) 2.2\%), edad (18 - 25 años, 26.5\%; 26 - 34 años, 26.5\%; 35 - 45 años, $24.2 \%$ y 46 o más años, $22.7 \%$ ) y ocupación de los entrevistados (obreros y empleados, $23.4 \%$; estudiantes, 18.8\%; hogar, 15.9\%; auto - empleados y empresarios, $14.8 \%$; técnicos y vendedores, $7.6 \%$; ejecutivos y gerentes, $6 \%$; desempleados y jubilados, $3.4 \%$; otras ocupaciones, $10.2 \%)$. En cuanto a su comportamiento de consumo La mayoría de los entrevistados (74.1\%) ya habían visitado con anterioridad la ciudad, mientras que solamente un $25.7 \%$ se visitantes lo hacían por primera vez. La distribución según las noches de estancia se manifestó de la siguiente manera: 3 noches o menos, 39.3\%; 4 noches 18.8\%; 5 a 6 noches, $21.4 \%$ y 7 noches o más, 20.5\%. La distribución del gasto turístico pudo establecerse en 3 segmentos. Los turistas con bajo gasto (500 MXN = 28.30 EUR o menos) constituyeron un 42\%, los de gasto medio (501 MXN a $1000 \mathrm{MXN}$ = 28.53 EUR a 56.59 EUR) un 37.6\% y los de alto gasto (1001 MXN = 56.65 EUR o más), un 20.4\%

La mayoría de los participantes de la muestra final fueron mexicanos, un $15.9 \%$ provenientes del vecino estado de Tamaulipas, mientras que un 10.6\% manifestó tener su lugar 
de residencia en el vecino estado de Coahuila. En cuotas superiores al $5 \%$ del total de la muestra se encontraron los visitantes originarios de entidades como el Distrito Federal (9.2\%), México (6.3\%), Guanajuato (6.2\%), Chihuahua (5.7\%) y Jalisco (5.1\%). En lo que respecta al turismo extranjero que participó en la muestra, estuvieron principalmente estadounidenses (2.4\%) y visitantes de otros países (4\%). Entre los entrevistados destacó un $1.2 \%$ de nuevoleoneses provenientes de lugares fuera del área metropolitana de Monterrey. La mayor parte pernoctaron en casa de familiares o amigos (51.5\%) seguidos por aquellos que utilizaron alojamientos comerciales con un $44.2 \%$ Un $4.2 \%$ de los entrevistados dijeron hospedarse en casa de su propiedad, mientras que sólo un caso no dio respuesta.

Respecto a las fuentes de información con mayor utilidad en la decisión de viajar a Monterrey La mayor parte de los entrevistados (62.9\%) se valieron de recomendaciones de amigos o parientes, mientras que un $12 \%$ dijo haber tomado la decisión gracias a la publicidad en medios masivos. Un 8.5 y un $7 \%$ lo hicieron por la información obtenida en páginas web y redes sociales. Mientras que un $5 \%$ dijo haber recurrido a otras fuentes y un $2.1 \%$ lo hizo gracias a la información en prensa.

\subsection{Proceso de análisis y resultados}

Se utilizó regresión por PLS para una reducción de dimensiones del conjunto de variables. Cada indicador y constructo recibió una etiqueta distintiva: MO_01 a MO_12 como indicadores de la variable motivación de viaje (MOTIV); IM_01 a IM_04 como indicadores de la imagen afectiva (IMG AFE), IM_05 a IM_20 como indicadores de la imagen cognitiva (IMG COG) y SA como único indicador de la satisfacción del turista (SAT). A diferencia de la regresión de componentes principales (donde no hay garantía de que las variables obtenidas sean pertinentes para explicar una variable dependiente) la regresión de PLS identifica los componentes principales de la variable independiente que también sean los más adecuados para explicar la variable dependiente (Mateos-Aparicio, 2011).

Una vez estimados los parámetros del modelo a través del software de análisis estadístico Smart PLS 2.0, se identificaron los componentes principales de las variables. En el caso de la variable motivación de viaje se identificaron tres componentes todos ellos de tipo atracción (pull). En el caso de la imagen del destino se identificaron cuatro componentes de imagen afectiva y cuatro de imagen cognitiva, mientras que la satisfacción del turista se mantuvo con su único indicador (Tabla 4). Como criterio se desecharon todos aquellos indicadores cuya carga o peso estuviera por debajo de 0.700

Tabla 4. Componentes principales identificados en las variables, pesos de sus indicadores y coeficientes de confiabilidad $\alpha$ de Cronbach.

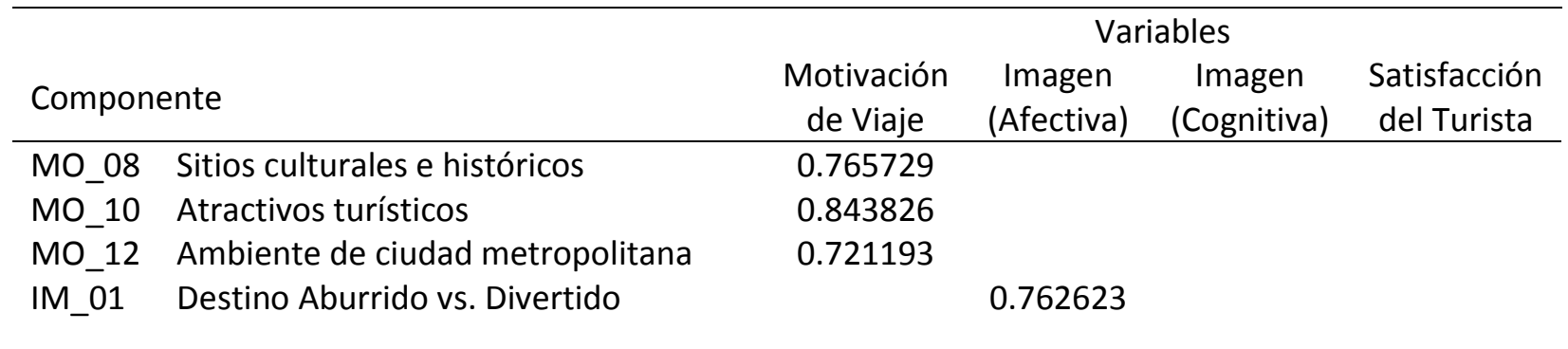




\begin{tabular}{|c|c|c|c|c|c|}
\hline IM_02 & Destino No Placentero vs. Placentero & & 0.841020 & & \\
\hline IM_03 & Destino Deprimente vs. Emocionante & & 0.772959 & & \\
\hline IM_04 & Destino Estresante vs. Relajante & & 0.711314 & & \\
\hline IM_05 & Desarrollo de infraestructura & & & 0.745192 & \\
\hline IM_07 & Servicios Turísticos & & & 0.739865 & \\
\hline IM_17 & Carácter familiar & & & 0.751822 & \\
\hline IM_20 & Belleza de paisajes & & & 0.778377 & \\
\hline \multirow[t]{2}{*}{ SA } & Satisfacción del Turista & & & & 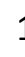 \\
\hline & $\alpha$ de Cronbach & 0.673554 & 0.775681 & 0.747133 & 1 \\
\hline
\end{tabular}

Fuente: Elaboración propia

El siguiente paso fue elaborar la expresión gráfica de la relación de variables sujeta de contraste a través de un diagrama de trayectoria (path diagram) y que se expresa en la figura 2.

Figura 2. Diagrama de trayectoria del modelo propuesto

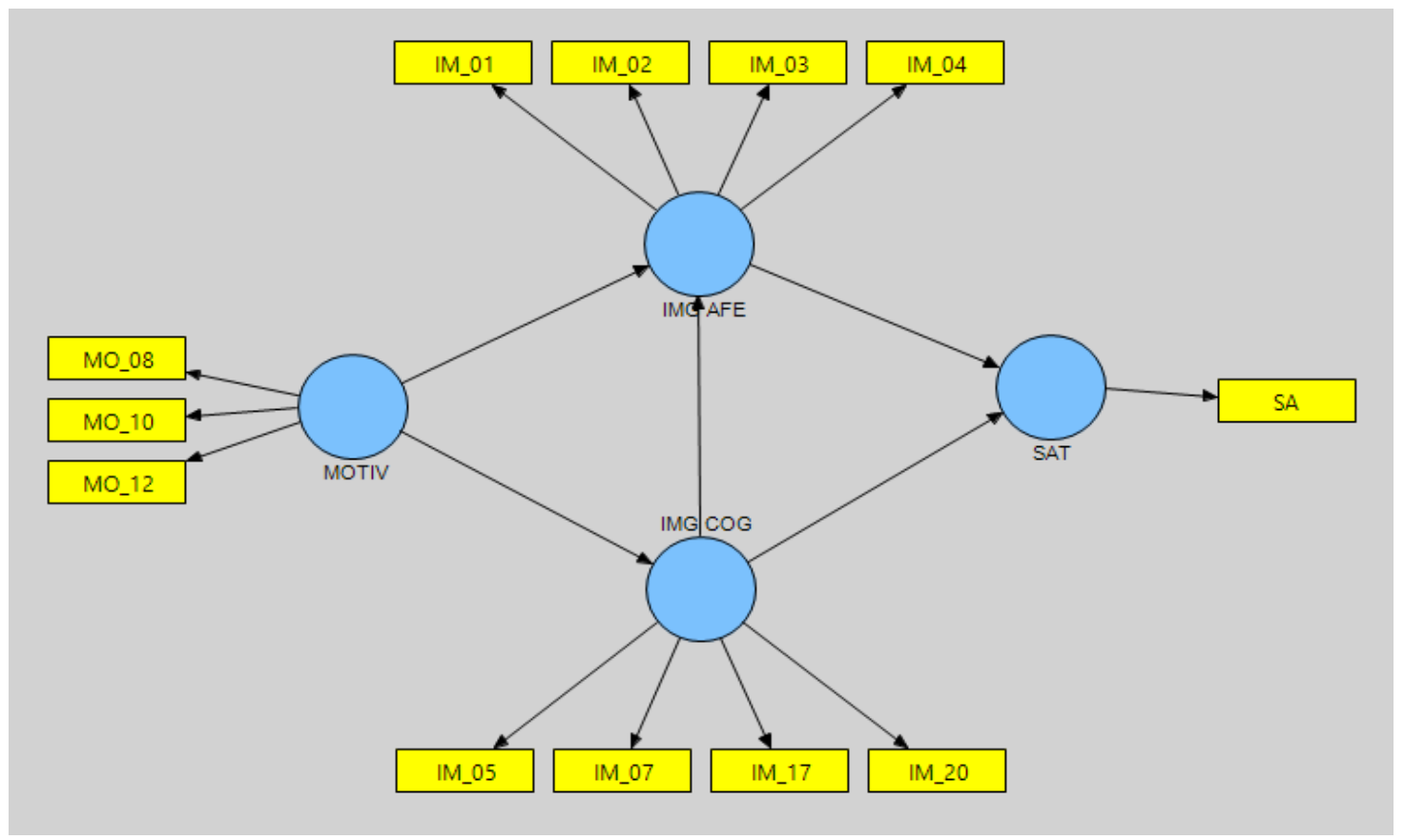

Fuente: Elaboración propia con el software Smart PLS 2.0

Una vez estimados los parámetros a través del software de análisis estadístico Smart PLS se procedió a la evaluación del modelo. Antes de proceder a la evaluación del modelo estructural se evaluó el modelo de medición.

Los valores superiores a 0.50 de la Varianza Promedio Extraída (AVE, por sus siglas en inglés de Average Variance Extracted), indicaron que los constructos eran válidos a nivel convergente. (Tabla 5) 
El efecto de la motivación de viaje sobre la satisfacción del turista a través de las dimensiones de la imagen de destino: El caso del turismo urbano de ocio a Monterrey, México.

Tabla 5. Varianza Promedio Extraída (AVE) de los constructos del modelo

\begin{tabular}{lc}
\hline Constructo & Varianza Promedio Extraída (AVE) \\
\hline Motivación de Viaje & 0.606168 \\
Imagen Afectiva & 0.598085 \\
Imagen Cognitiva & 0.568454 \\
Satisfacción del Turista & 1.000000 \\
\hline
\end{tabular}

Fuente: Elaboración propia utilizando el software Smart PLS 2.0

La revisión de la tabla de cargas cruzadas de los constructos, donde todos los ítems cargaban con su respectivo constructo, arrojó evidencia para la validez discriminante del modelo en sus ítems (Tabla 6).

Tabla 6. Cargas cruzadas de los indicadores de los constructos

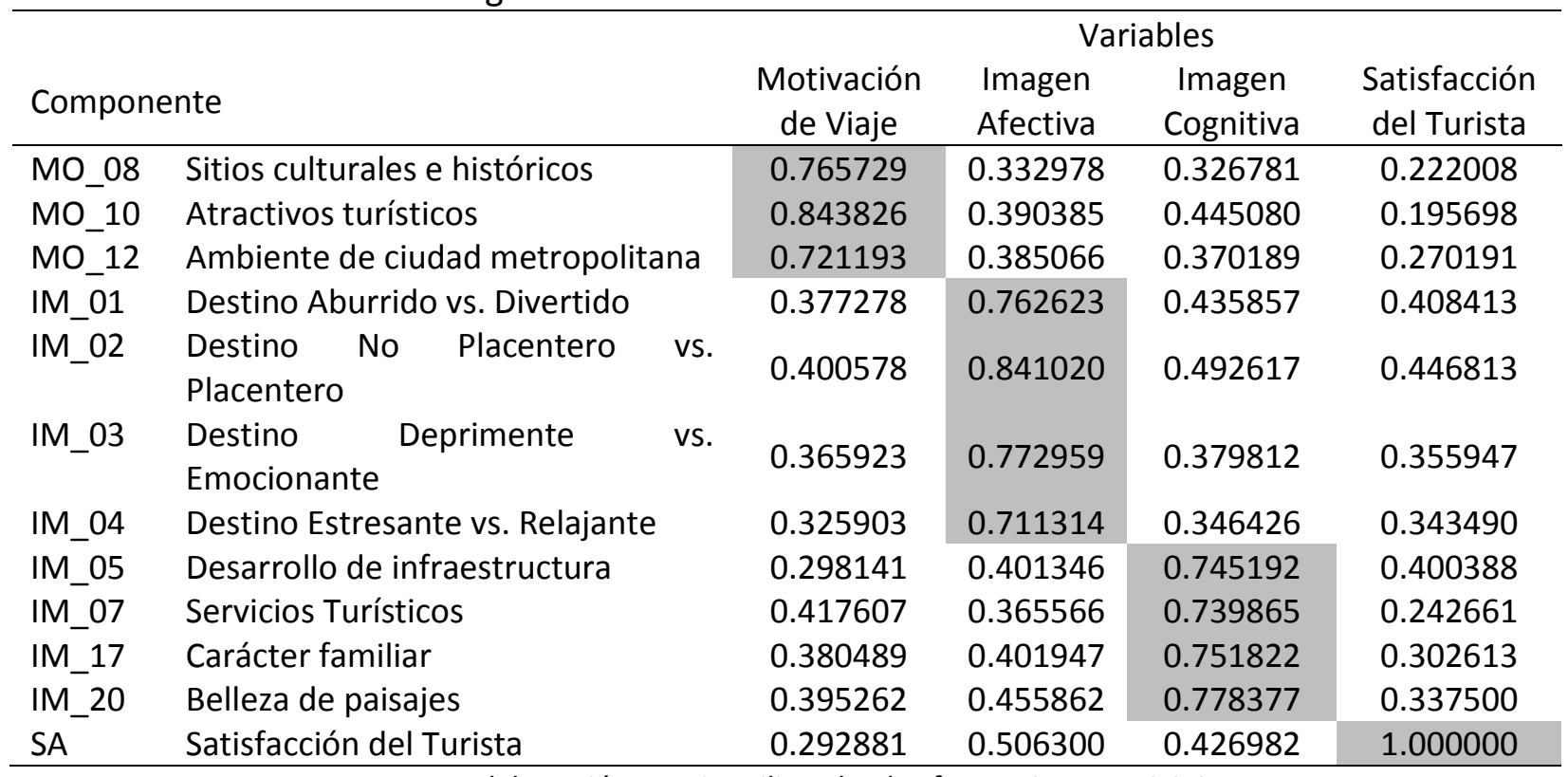

Fuente: Elaboración propia utilizando el software Smart PLS 2.0

Para comprobar la validez discriminante del modelo de medición se comparó la raíz cuadrada de la AVE con la correlación entre las variables del modelo. Al resultar superior la raíz cuadrada en todos los casos se asume que el modelo es válido de forma discriminante (Tabla 7).

Tabla 7. Comprobación de la validez discriminante a nivel constructo

\begin{tabular}{lccccc}
\hline Constructo & $\begin{array}{c}\text { Raíz } \\
\text { Cuadrada } \\
\text { de la AVE }\end{array}$ & $\begin{array}{c}\text { Imagen } \\
\text { Afectiva }\end{array}$ & $\begin{array}{c}\text { Imagen } \\
\text { Cognitiva }\end{array}$ & $\begin{array}{c}\text { Motivación } \\
\text { de Viaje }\end{array}$ & $\begin{array}{c}\text { Satisfacción } \\
\text { del Turista }\end{array}$ \\
\hline Imagen Afectiva & 0.77336 & 1 & & & \\
Imagen Cognitiva & 0.75396 & 0.540526 & 1 & 1 & 1 \\
Motivación de Viaje & 0.77857 & 0.476755 & 0.494090 & 0.292881 & 1 \\
Satisfacción del Turista & 1 & 0.506300 & 0.426982 & \\
\hline \multicolumn{7}{c}{ Elaboración propia }
\end{tabular}


La confiabilidad del modelo de medición a nivel ítem se comprobó dados los valores de cargas de los ítems en su respectivo constructo, superiores todos ellos a 0.50 mientras que la confiabilidad a nivel constructo se evidenció vía la Confiabilidad Compuesta (Composite Reliability) superior a 0.60 (Tabla 8).

Tabla 8. Confiabilidad compuesta de los constructos del modelo

\begin{tabular}{lc}
\hline Constructo & Confiabilidad Compuesta \\
\hline Imagen Afectiva & 0.855723 \\
Imagen Cognitiva & 0.840433 \\
Motivación de Viaje & 0.821361 \\
Satisfacción del Turista & 1.000000 \\
\hline
\end{tabular}

Fuente: Elaboración propia utilizando el software Smart PLS 2.0

Se procedió entonces a la evaluación del modelo interior para poner a prueba las hipótesis establecidas en el diagrama de trayectorias inicialmente planteado (Figura 2). Los criterios utilizados para evaluar un modelo interior implican la revisión del coeficiente de determinación $\mathrm{R}^{2}$, los coeficientes de trayectoria estandarizados, los estadísticos - t y los niveles de significancia. Un resumen de indicadores puede observarse en la tabla 9.

Tabla 9. Resultados significativos del modelo estructural

\begin{tabular}{|c|c|c|c|c|c|c|}
\hline & Relación & & $\begin{array}{c}\text { Efecto } \\
\text { propuesto }\end{array}$ & $\begin{array}{c}\text { Coeficiente de } \\
\text { Trayectoria }\end{array}$ & Estadístico t & $\begin{array}{c}\text { Nivel de } \\
\text { significancia }\end{array}$ \\
\hline $\mathrm{H}_{1}$ & $\begin{array}{l}\text { Motivación de Viaje } \\
\text { Imagen Afectiva }\end{array}$ & $\rightarrow$ & + & 0.277409 & 2.430311 & $p<0.025$ \\
\hline $\mathrm{H}_{2}$ & $\begin{array}{l}\text { Motivación de Viaje } \\
\text { Imagen Cognitiva }\end{array}$ & $\rightarrow$ & + & 0.494090 & 5.343098 & $p<0.0001$ \\
\hline $\mathrm{H}_{3}$ & $\begin{array}{l}\text { Imagen Cognitiva } \rightarrow \text { Imag } \\
\text { Afectiva }\end{array}$ & & + & 0.403461 & 3.270760 & $p<0.0005$ \\
\hline $\mathrm{H}_{4}$ & $\begin{array}{l}\text { Imagen Cognitiva } \\
\text { Satisfacción del Turista }\end{array}$ & $\rightarrow$ & + & 0.216596 & 1.865113 & $p<0.05$ \\
\hline $\mathrm{H}_{5}$ & $\begin{array}{l}\text { Imagen Afectiva } \\
\text { Satisfacción del Turista }\end{array}$ & $\rightarrow$ & + & 0.389224 & 3.625045 & $p<0.0005$ \\
\hline
\end{tabular}

Fuente: Elaboración propia utilizando el software Smart PLS 2.0

Las estadísticas finales del modelo propuesto pueden observarse en la tabla 10. Los coeficientes $\mathrm{R}^{2}$ confirman los efectos determinantes de la motivación del turista sobre los componentes (afectivo y cognitivo) de la imagen de destino, así como el efecto determinante de la imagen cognitiva sobre la imagen afectiva. Por último se confirma el efecto causal de ambos componentes sobre la satisfacción del turista estableciéndose así como variables mediadoras del efecto de la motivación de viaje sobre la satisfacción. 
Tabla 10. Estadísticas finales del modelo propuesto

\begin{tabular}{lcccccc}
\hline Constructo & AVE & CC & $\mathrm{R}^{2}$ & $\alpha$ & Com. & Red. \\
\hline $\begin{array}{l}\text { Imagen } \\
\text { Afectiva }\end{array}$ & 0.598085 & 0.855723 & 0.350338 & 0.775681 & 0.598085 & 0.162208 \\
$\begin{array}{l}\text { Imagen } \\
\text { Cognitiva }\end{array}$ & 0.568454 & 0.840433 & 0.244124 & 0.747133 & 0.568454 & 0.139119 \\
$\begin{array}{l}\text { Motivación de } \\
\text { Viaje }\end{array}$ & 0.606168 & 0.821361 & - & 0.673554 & 0.606168 & - \\
$\begin{array}{l}\text { Satisfacción } \\
\text { del Turista }\end{array}$ & 1 & 1 & 0.289546 & 1 & 1 & 0.242633 \\
\hline
\end{tabular}

AVE $=$ Varianza Promedio Extraída CC $=$ Confiabilidad Compuesta $\alpha=$ Coeficiente $\alpha$ de Cronbach

Com. $=$ Comunalidad Red. $=$ Redundancia

Fuente: Elaboración propia utilizando el software Smart PLS 2.0

\section{CONCLUSIONES}

Una ciudad es capaz de convertirse en un destino turístico de éxito en la medida que consiga atraer y fidelizar a sus visitantes. El camino más sencillo es satisfaciendo sus expectativas y propiciando su lealtad. Los gestores de destino, apoyados por el método científico, pueden obtener información de primera calidad a través de la aplicación de técnicas de análisis que les permitan relacionar las variables que condicionan la conducta de sus turistas. En el caso de Monterrey, N.L., la metodología propuesta por este trabajo permitió identificar los aspectos estadísticamente significativos que más pesan para el turista dando certidumbre a la información para la toma de decisiones estratégicas de marketing y gestión turística.

Con respecto a la motivación se identificó que los tres principales factores de atracción a la ciudad (en función de su imagen) son los sitios culturales e históricos, los atractivos turísticos y el ambiente de ciudad metropolitana. No es de sorprenderse que una ciudad como Monterrey genere en la mente de los turistas expectativas sobre sitios para conocer dado que es una de las ciudades más grandes del país. Sus visitantes, esperan encontrar en ella una multitud de opciones para recorrer y visitar. La motivación es un poderoso medio de segmentación. Los gestores de la ciudad pueden dirigir su oferta hacia los turistas provenientes de ciudades emisoras que no poseen el carácter de gran ciudad que tiene Monterrey con la seguridad de que aquellos atractivos y sitios más relevantes de su patrimonio gozan de una ventaja al enmarcarse en un contexto de metrópoli.

Por su parte, este estudio permitió concluir que la imagen que la ciudad genera en la mente de los turistas sigue las características planteadas en la literatura del tema. Los turistas que visitan la ciudad desarrollan determinadas emociones y también configuran su retrato de la ciudad en base a determinadas características. En el caso de la muestra estudiada, los turistas perciben a la ciudad en función de su capacidad para divertirlos, emocionarlos, hacerlos sentir cómodos y relajados. Asimismo, los componentes de la imagen cognitiva ofrecen un panorama sobre la ciudad centrado en su desarrollo de infraestructura, los servicios disponibles para los turistas, su carácter familiar todo ello enmarcado por bellos paisajes. Identificar dichos componentes a través de un modelo estructural ajustado por mínimos cuadrados parciales (PLS) 
es particularmente útil ya que a nivel estadístico dicha configuración de imagen se da en función de los motivadores antes señalados.

La congruencia funcional entre motivaciones, dimensiones de la imagen percibida y satisfacción permiten una configuración sólida de estrategias de marketing orientadas hacia el segmento familiar y buscador de actividades propias de una gran ciudad. No es de extrañarse que la valoración de los turistas en el sentido cognitivo despierte en el turista hacia Monterrey toda clase de emociones positivas por lo que la estrategia ideal a seguir por el destino estaría centrada en asociar el carácter de gran ciudad moderna y bien equipada con un entorno bello llena de servicios dirigidos hacia el entretenimiento y emoción familiar a la vez que cómoda y libre de preocupaciones. Todo ello como factores que abonarían a que el turista se lleve una experiencia satisfactoria de su visita al destino.

Este estudio realizó un abordaje sencillo de la relación entre variables que forman la conducta del turista. Sin embargo, son muchas otras variables las que pueden estudiarse para generar indicadores fiables y adecuados a su naturaleza compleja. Valor, expectativas, calidad en servicios, son algunos otros constructos que pueden constituirse en opciones para configurar modelos que den respuesta a las grandes interrogantes que subsisten sobre factores claves para detonar la competitividad de los destinos mexicanos configurando su oferta en función de la demanda. Modelos estructurales vía PLS son pues una herramienta adecuada, válida y fiable para el análisis de la realidad turística de cara a la necesidad de contar con bases para la toma de decisiones. Dichos instrumentos e información resultarán valiosos para la obtención de ventajas competitivas cimentadas en el aprovechamiento del conocimiento sobre la dinámica de la satisfacción turística, permitiendo obtener mejores resultados de gestión.

\section{REFERENCIAS BIBLIOGRÁFICAS}

Anderson, E. (1996). Customer satisfaction and price tolerance. Marketing Letters, 7(3), 265-274. Anderson, E. W., y Sullivan, M. W. (1993). The antecedents and consequences of customer satisfaction for firms. Marketing Science, 12(2), 125-143.

Anderson, E., Fornell, C., y Mazvancheryl, S. K. (2004). Customer satisfaction and shareholder value. Journal of Marketing(4), 172-185.

Anderson, W., Juaneda, C., y Sastre, F. (2009). Influences of pro- all-inclusive travel decisions. Tourism Review, 64(2), 4-18.

Asher, M. (1989). Measuring customer satisfaction. The TQM Magazine, 1(2), 93-96.

Ashworth, G. (2012). Do we understand urban tourism? Journal of Tourism Hospitality, 1(4).

Baloglu, S., y McCleary, W. (1999). U.S. international pleasure travelers' images of four Mediterranean destinations: a comparison of visitors and nonvisitors. Journal of Travel Research, 38(2), 144 - 152.

Baloglu, S., Pekcan, A., Chen, S. L., y Santos, J. (2004). The relationship between destination performance, overall satisfaction, and behavioral intention for distinct segments. Journal of Quality Assurance in Hospitality y Tourism, 4(3-4), 149-165.

Bansalt, H., y Eiselt, H. A. (2004). Exploratory researchof tourist motivations and planning. Tourism Management, 25, 387-396. 
Battour, M., Ismail, M. N., y Battor, M. (2014). Islamic tourism: an empirical examination of travel motivation and satisfaction in Malaysia. Current Issues in Tourism, 1-18.

Beerli, A., y Martín, J. D. (2004). Factors influencing destination image. Annals of Tourism Research, 31(1), 657 - 681.

Beerli, A., y Martín, J. D. (2004). Tourists' characteristics and the perceived image of tourist destinations: a quantitative analysis - a case study of Lanzarote, Spain. Tourism Management (25), 623-636.

Bernini, C., y Cagnone, S. (2012). Analysing tourist satisfaction at a mature and multi-product destination. Current Issues in Tourism.

Bigné, J. E., Sánchez, M. I., y Sánchez, J. (2001). Tourism image, evaluation variables and after purchase behaviour: inter-relationship. Tourism Management (22), 607 - 616.

Buhalis, D. (2000). Marketing the competitive destination of the future. Tourism Management(21), 97 - 116.

Castaño Blanco, J. M., Moreno Sáez, A., García Dauder, S., y Crego Díaz, A. (2003). Aproximación psicosocial a la motivación turística: variables implicadas en la elección de Madrid como destino. Estudios Turísticos(158), 5-41.

Chan, J. L., y Baum, T. (2007). Motivation factors of ecotourists in ecolodge accomodation: The push and pull factors. Asia Pacific Journal of Tourism Research, 12(4), 349-364.

Chen, C.-F., y Tsai, D. (2007). How destination image and evaluative factors affect behavioral intentions? Tourism Management, 28, 1115 - 1122.

Chen, Y., Li, R., y Chen, S. (2013). Relationships among adolescents' leisure motivation, leisure involvement, and leisure satisfaction: A structural equation model. Social Indicators Research, 110(3), 1187-1199.

Chi, C. G.-Q., y Qu, H. (2008). Examining the structural relationships of destination image, tourist satisfaction and destination loyalty: An integrated approach. Tourism Management(29), 624636.

Chon, K. (1989). Understanding recreational traveller's motivation, attitude and satisfaction. The Tourist Review, 1, 3-7.

Chung, J. Y., y Petrick, J. F. (2012). Measuring attribute - specific and overall satisfaction with destination experience. Asia Pacific Journal of Tourism Research.

Correia, A., Kozak, M., y Ferradeira, J. (2013). From tourist motivation to tourist satisfaction. International Journal of Culture, Tourism and Hospitality Research, 7(4), 411 - 424.

Crompton, J. L. (1979). Motivation for pleasure vacation. Annals of Tourism Research, 6, 408-424.

Crompton, J. L., y McKay, S. L. (1997). Motives of visitors attending festival events. Annals of Tourism Research, 24(2), 425-439.

Dann, G. S. (1977). Anomie, ego-enhancement and tourism. Annals of Tourism Research (4), 184194.

DATATUR. (11 de noviembre de 2014). Sistema de Información Estadística del Sector Turístico de México. Obtenido de Compendio Estadístico del Sector Turismo en México: http://www.datatur.sectur.gob.mx/SitePages/CompendioEstadistico.aspx

Devesa, M., Laguna, M., y Palacios, A. (2010). The role of motivation in visitor satisfaction: Empirical evidence in rural tourism. Tourism Management, 31, 547-552. 
Dmitrovic, T., Cvelbar, L. K., Kolar, T., Brencic, M., Ograjensek, I., y Zabkar, V. (2009). Conceptualizing tourist satisfaction at the destination level. International Journal of Culture, Tourism and Hospitality Research, 3(2), 116-126.

Dwyer, L., y Kim, C. (2003). Destination competitiveness: determinants and indicators. Current Issues in Tourism, 6(5), 369 - 414.

Echtner, C. M., y Ritchie, J. B. (2003). The meaning and measurement of destination image. The Journal of Tourism Studies, 37 - 48.

Edwards, D., Griffin, T., y Hayllar, B. (2008). Urban tourism research: developing an agenda. Annals of Tourism Research, 35(4), 1032-1052.

Enright, M., y Newton, J. (2004). Tourism destination competitiveness: a quantitative approach. Tourism Management (25), 777 - 788.

Fallon, P., y Schofield, P. (2006). The dynamics of destination attribute importance. Journal of Business Research (59), 709 - 713.

Faullant, R., Matzler, K., y Füller, J. (2008). The impact of satisfaction and image on loyalty: the case of Alpine ski resorts. Managing Service Quality, 18(2), 163-178.

Galdini, R. (2007). Tourism and the City: Opportunity for Regeneration. Tourismos: An International Multidisciplinary Journal of Tourism, 2(2), 95-111.

Gnoth, J. (1997). Tourism motivation and expectation formation. Annals of Tourism Research, 24(2), 283-304.

Gómez, M., Molina, A., y Esteban, A. (2013). What are the main factors attracting visitors to wineries? A PLS multi-group comparison. Quality y Quantity, 47(5), 2637-2657.

Guerrero González, P., y Ramos Mendoza, J. R. (2011). Introducción al Turismo. México, D.F.: Patria.

Gunn, C. (1988). Vacationscapes: Designing tourist regions. New York: Van Nostrand Reinhold.

Hallowell, R. (1996). The relationships of customer satisfaction, customer loyalty, and profitability: an empirical study. International Journal of Service Industry Management, 7(4), 27-42.

Henlein, M., y Kaplan, A. (2004). A beginner's guide to partial least squares analysis. Understanding Statistics, 3(4), 283-297.

Homburg, C., Koschate, N., y Hoyer, W. D. (2005). Do satisfied customers really pay more? A study of the relationship between customer satisfaction and willingness to pay. Journal of Marketing, 69(2), 84-96.

Hsu, C. H., Cai, L. A., y Li, M. (2010). Expectation, motivation, and attitude: A tourist behavioral model. Journal of Travel Research, 49(3), 282-296.

Huang, S., y Hsu, C. H. (2009). Travel motivation: linking theory to practice. International Journal of Culture, Tourism and Hospitality Research, 3(4), 287-295.

Huber, F., Herrmann, A., y Wricke, M. (2001). Customer satisfaction as an antecedent of price acceptance: results of an empirical study. Journal of Product y Brand Management, 160-169.

Hull, C. L. (1943). Principles of Behavior. New York: Appleton-Century-Croft.

Hultman, M., Kazeminia, A., y Ghasemi, V. (2015). Intention to visit and willingness to pay premium for ecotourism: The impact of attitude, materialism, and motivation. Journal of Business Research, 68, 1854-1861.

INEGI. (2010). Instituto Nacional de Estadística y Geografía. Recuperado el 25 de agosto de 2015, de

Cuéntame:

Investigaciones Turísticas 126

$\mathrm{N}^{\circ} 14$, julio-diciembre 2017, pp. 109-129 
http://cuentame.inegi.org.mx/monografias/informacion/NL/Poblacion/default.aspx?tema= MEye=19

Iso-Ahola, S. (1982). Towards a social psychology of tourist motivation: A rejoinder. Annals of Tourism Research(9), 256-261.

ITB Berlín. (Diciembre 2014). ITB World Travel Trends Report. Berlín: Messe Berlin GmbH.

Ji, M., Wong, I. A., Eves, A., y Scarles, C. (2016). Food-related personality traits and the moderating role of novelty-seeking in food satisfaction and travel outcomes. Tourism Management, 57, 387-396.

Kao, M., Patterson, I., Scott, N., y Li, C. K. (2008). Motivations and satisfactions of taiwanese tourists who visit Australia. Journal of Travel and Tourism Marketing, 24(1), 17 - 33.

Khaki, A., y Sahaf, M. (2011). Satisfaction of tourists visiting the Kashmir Division. South Asian Journal of Tourism and Heritage, 4(1), 63-71.

Kim, K. (2008). Analysis of structural equation model for the student pleasure travel market: Motivation, involvement, satisfaction, and destination loyalty. Journal of Travel y Tourism Marketing, 24(4), 297-313.

Kim, N., y Chalip, L. (2004). Why travel to the FIFA world cup? effects of motives, background, interest, and constraints. Tourism Management, 25(6), 695-707.

Kotler, P., Haider, D., y Rein, I. (1994). Mercadotecnia de Localidades: cómo atraer inversiones, industrias y turismo a ciudades, regiones, estados y países. México: Diana.

Law, C. M. (1992). Urban Tourism and its Contribution to Economic Regeneration. Urban Studies, 29(3/4), 599-618.

Lee, C.-K., Lee, Y.-K., y Wicks, B. E. (2004). Segmentation of festival motivation by nationality and satisfaction. Tourism Management, 25(1), 61-70.

Lee, T. H. (2009). A structural model to examine how destination image, attitude, and motivation affect the future behavior of tourists. Leisure Sciences: An Interdisciplinary Journal, 31(3), 215-236.

Lee, T., y Hsu, F. (2013). Examining how attending motivation and satisfaction affects the loyalty for attendees at Aboriginal festivals. International Journal of Tourism Research, 15(1), 18-34.

Leyva, O., y Olague, J. T. (2014). Modelo de ecuaciones estructurales por el método de mínimos cuadrados parciales (Partial Least Squares). En K. Sáenz-López, y G. Tamez-González (Edits.), Métodos y técnicas cualitativas y cuantitativas aplicables a la investigación en ciencias sociales (págs. 479-497). México, D.F.: Tirant Humanidades.

Lubbe, B. (1998). Primary image as a dimension of destination image: An empirical assessment. Journal of Travel and Tourism Marketing, 7(4), 21-43.

Maslow, A. H. (1943). A theory of human motivation. Psychological Review (50), 370-396.

Maslow, A. H. (1970). Personality and motivation. Nueva York: Harper and Row.

Mateos-Aparicio, G. (2011). Partial least squares (PLS) methods: Origins, evolution, and application to social sciences. Communications in Statistics, 40(13), 2305-2317.

Matos, N., Mendes, J., y Valle, P. (2012). Revisiting the destination image construct through a conceptual model. Dos Algarves. A multidisciplinary e-journal (21), 101-117.

Medina, C., Rey, M., y Rufín, R. (2010). Imagen de los destinos turísticos urbanos y lealtad del turista... ¿Actitud o comportamiento? Estudios y Perspectivas en Turismo, 19, 279-298. 
Mehmetoglu, M., y Normann, $\varnothing$. (2013). The link between travel motives and activities in naturebased tourism. Tourism Review, 68(2), 3-13.

Meng, F., Tepanon, Y., y Uysal, M. (2008). Measuring tourist satisfaction by attribute and motivation: The case of a nature-based resort. Journal of Vacation Marketing, 14(41), 41-56.

Nicolau, J. L., y Más, F. J. (2006). The influence of distance and prices on the choice of tourist destinations: The moderating role of motivations. Tourism Management, 27, 982-996.

Nunkoo, R., y Ramkissoon, H. (2012). Structural equation modelling and regression analysis in tourism research. Current Issues in Tourism, 15(8), 777-802.

Nyaupane, G. P., Paris, C. M., y Teye, V. (2011). Study abroad motivations, destination selection and pre-trip attitude formation. International Journal of Tourism Research, 13(3), 205-217.

Organización Mundial del Turismo. (1985). Identification and evaluation of those components of tourism which have a bearing on tourist satisfaction and which can be regulated and state measures to ensure adequate quality of tourism services. Madrid: OMT.

Organización Mundial del Turismo. (2014). Panorama OMT del Turismo Internacional. Madrid: UNWTO.

Paskaleva-Shapira, K. A. (2007). New Paradigms in City Tourism Management: Redefining Destination Promotion. Journal of Travel Research, 46, 108-114.

Pearce, P. L. (1988). The Ulysses Factor: Evaluating Visitors in Tourist Settings. Nueva York: Springer-Verlag.

Pearce, P. L. (1993). Fundamentals of tourist motivation. En D. Pearce, y R. Butler (Edits.), Tourism Research: Critiques and Challenges (págs. 85-105). Londres: Routledge y Kegan Paul.

Pearce, P. L. (2005). Tourist Behavior. Themes and Conceptual Schemes. Clevedon: Channel View Publications.

Phetvaroon, K., Rachjaibun, N., y Promsivapallop, P. (2006). Tourist satisfaction with tourism product in Phuket, Thailand. En I. Assenov (Ed.), The Fifht Asia Pacific Forum for Graduate Student Research in Tourism Threats and Challenges to the Tourism Industry: Reform and Perform, (págs. 315-324). Bangkok.

Propin Frejomil, E., y Sánchez Crispín, Á. (2002). La estructura regional del turismo en México. Ería, 59, 386-394.

Propin Frejomil, E., y Sánchez Crispín, Á. (2007). Tipología de los destinos turísticos preferenciales en México. Cuadernos de Turismo (19), 147-166.

Rogerson, C. M. (2013). Urban tourism, economic regeneration and inclusion: Evidence from South Africa. Local Economy, 28(2), 188-202.

Rust, R. T., y Zahorik, A. J. (1993). Customer satisfaction, customer retention, and market share. Journal of Retailing, 69(2), 193-215.

Ryan, C., y Glendon, I. (1998). Application of leisure motivation scale to tourism. Annals of Tourism Research, 25(1), 169-184.

San Martín, H., y Rodríguez, I. A. (2008). Exploring the cognitive-affective nature of destination image and the role of psychological factors in its formation. Tourism Management, 29(2), 263-277.

Sánchez Crispín, Á., López López, Á., y Propin Frejomil, E. (2005). Estructura territorial del turismo en la Zona Metropolitana de Monterrey. Investigaciones Geográficas (058), 80-105. 
Smith, S., Costello, C., y Muenchen, R. (2010). Influence of push and pull motivations on satisfaction and behavioral intentions within a culinary tourism event. Journal of Quality Assurance in Hospitality y Tourism (11), 17-35.

Stabler, W. J. (1988). The image of destination regions: theoretical and empirical aspects. En B. Goodall, y G. Ashworth (Edits.), Marketing in the Tourism Industry: the Promotion of Destination Regions (págs. 133-161). London: Croom Helm.

Tasci, A. D., Gartner, W. C., y Cavusgil, S. T. (2007). Conceptualization and operationalization of destination image. Journal of Hospitality y Tourism Research (34), 194 - 223.

Varasteh, H., Marzuki, A., y Rasoolimanesh, S. M. (2015). Factors affecting international students' travel behavior. Journal of Vacation Marketing, 21(2), 131-149.

Violier, P., y Zárate Martín, M. A. (2007). Turismo urbano y políticas para su gestión en Francia y España. Estudios Geográficos, 68(262), 321-347.

Vroom, V. H. (1964). Work and Motivation. New York: Wiley.

Wang, S. Y., y Hsu, M. (2010). The relationships of destination image, satisfaction, and behavioral intentions: An integrated model. Journal of Travel y Tourism Marketing, 27(8), 829-843.

Wang, S., y Qu, H. (2006). A study of tourists' satisfaction determinants in the context of the Pearl River Delta sub-regional destinations. Journal of Hospitality y Leisure Marketing, 14(3), 4963.

Wong, I. A., y Tang, S. L. (2016). Linking travel motivation and loyalty in sporting events: The mediating roles of event involvement and experience, and the moderating role of spectator type. Journal of Travel y Tourism Marketing, 33(1), 63-84.

Wu, K., Raab, C., Chang, W., y Krishen, A. (2016). Understanding chinese tourists' food consumption in the United States. Journal of Business Research, 69(10), 4706-4713.

Xiao, G. (2007). Urban Tourism: Global - Local Relationships in Dalian, China. Tesis Doctoral. Waterloo, Canadá: University of Waterloo.

Yoon, Y., y Uysal, M. (2005). An examination of the effects of motivation and satisfaction on destination loyalty: a structural model. Tourism Management (26), 45-56.

\section{AGRADECIMIENTOS}

Los autores expresan su agradecimiento a la Corporación para el Desarrollo Turístico de Nuevo León, México (CODETUR NL) por el apoyo recibido en la realización de esta investigación. Asimismo, a los revisores de la revista por sus valiosas aportaciones. 